\title{
Dynamics of nested, self-similar winnerless competition in time and space
}

\author{
Maximilian Voit and Hildegard Meyer-Ortmanns* \\ Physics and Earth Sciences, Jacobs University Bremen, P.O. Box 750561, 28725 Bremen, Germany
}

(Received 29 January 2019; revised manuscript received 27 May 2019; published 6 September 2019)

\begin{abstract}
We construct $n$ levels of nested, self-similar winnerless competition dynamics of which we explicitly work out the first three levels in the framework of generalized Lotka-Volterra equations. We choose microscopic rules such that the competition in the form of rock-paper-scissors is played between metapopulations, populations, and individuals at the same time. The trajectory of individual activities moves through a hierarchically structured heteroclinic network in a desired way. The hierarchy in structure is able to induce a separation of timescales that translates into nested spirals if the heteroclinic networks are coupled via diffusion on a spatial grid. For sufficiently strong diffusion the dynamics of interacting heteroclinic networks gets synchronized between the sites, which amounts to a large dimensional reduction of phase space. Possible applications lie in ecology and in brain dynamics. Our model reproduces in particular chunking dynamics with slow oscillations modulating fast oscillations modulating faster ones as observed in brain dynamics.
\end{abstract}

DOI: 10.1103/PhysRevResearch.1.023008

\section{INTRODUCTION}

Processes related to information flow in the brain are characterized as dynamic, hierarchically nested, continuously changing [1,2], and self-similar [3]. Antagonistic tendencies for independence and cooperation coexist while information is created, maintained for some time, and dissipated thereafter. This leads to the formation of temporary and nested spatiotemporal coalitions among neuronal assemblies. The feature of being nested refers to the interlocking of processes that run simultaneously on different temporal and spatial scales. Characteristics of self-similarity are seen in power spectra of brain activity [4], in self-similar wirings [5], and in computational motifs at the micro-, meso-, and macroscales [3]. Brain dynamics proceeds via sequential segmentation of information that is manifest in sequences of electroencephalography (EEG) microstates [6], which are brief periods of stable scalp topography with a quasistationary configuration of the scalp potential field.

A mathematical framework with a network attractor between nodes that is supposed to represent the EEG microstates is provided by the dynamics of winnerless competition (WLC) (see [7-9] and references therein). Winnerless competition network activity comprises discrete sequential events. As an effective description it can be applied at all levels of temporal and spatial hierarchical organization, from motor and sensory processing to higher-level behavior and cognition. In relation to cognitive processes, these events may correspond to the coding of cognitive information, the temporary winners in

\footnotetext{
*h.ortmanns@jacobs-university.de

Published by the American Physical Society under the terms of the Creative Commons Attribution 4.0 International license. Further distribution of this work must maintain attribution to the author(s) and the published article's title, journal citation, and DOI.
}

a competitive information scenario. On a formal level, the "events" in WLC are described as saddles (with one or higherdimensional unstable manifolds), connected via heteroclinic orbits and forming heteroclinic sequences, cycles, or even whole networks as we will see later.

In brain dynamics such sequences of metastable states are observed on different timescales, ranging from milliseconds to seconds [10]. These sequences may be nested as reflected in so-called chunking dynamics [11] when, for example, slow oscillations of neuronal activity modulate fast oscillations modulate even faster ones. In general, chunking is a dynamical phenomenon observed in brain dynamics when long informational sequences are iteratively split into blocks with concatenated units inside the blocks. We will keep applications to brain dynamics in the focus of our interest, but before we should also point to a different range of applications of WLC, since we use the terminology from this range.

This is evolutionary game theory. In this context, rockpaper-scissors (RPS) games as a simple form of WLC have been extended towards more species $(N>3)$ and cyclic predation of $r>1$ prey, combined with possible reproduction and deletion processes as well as different realizations of mobility on a spatial grid. A large variety of fascinating, sometimes counterintuitive phenomena were then observed, based on simple microscopic rules (see [12,13] for reviews). The results are formulated in terms of neutral or competing self-organized alliances between subpopulations, whose individuals range from bacteria to socially acting agents. In particular, RPS games can emerge on a coarse scale [14] between three alliances of two species each. Conversely, in so-called $(6,3)$ games with six species cyclically predating on three other species, competing domains can form with RPS played inside these domains [15-17]. These works provide examples of nested, but not self-similar dynamics. Concretely, in Ref. [17], Labavić and Meyer-Ortmanns considered a set of rules with six species (corresponding to information items in cognitive dynamics), cyclically preying on three other species, 
which resulted in the formation of two rivaling domains on a coarse scale and RPS played inside the domains.

Against this background of game theory, it is natural to look for a modification of the set of microscopic rules so that RPS is also played on the coarse scale between the former domains and, moreover, iteratively on even larger scales with accordingly more species. If such a choice is possible, a single set of rules should lead to nested self-similar dynamics of WLC, here particularly realized as a "fractal" of RPS games. More generally, the question then arises of how to select the microscopic rules of the game to end up with a desired and preselected output in terms of preselected alliances or fights between competing species.

Although RPS is played on many space and time scales, usually these very different realizations do not arise from a single set of microscopic rules on a common lowest level of hierarchy that we are after here. From this point of view, the search for a fractal (in the physical sense) seems to be for curiosity, since we are not aware of examples where nested self-similar dynamics is realized to a higher order in the number of levels. On the other hand, because of this peculiar choice, it needs guiding principles to shortcut the search for finding appropriate rules of construction and to end up at a desired pattern of competition and temporary winners. This is in contrast to previous work, where the choice of the rules of the game together with deletion, reproduction, and diffusion processes were based on heuristic arguments and the forming patterns of alliances emerged in numerical simulations.

In this paper we demonstrate how to choose such a set of microscopic rules in a single set of WLC equations ${ }^{1}$ so that we observe both nested hierarchies in the temporal evolution (which is chunking dynamics with chunks corresponding to subsets of oscillating temporarily dominant items) and nested spatial patterns if the WLC units are coupled on a spatial grid. For simplicity, from now on we will use the language from ecological applications and call the temporary information items of cognitive processes, located at saddles in phase space, just species that are involved in games of competition.

\section{MODEL}

Towards a fractal of RPS, we start with the construction of an appropriate attractor at single sites of a spatial grid, in which the chasing of (sets of) species is implemented by heteroclinic connections and the temporary dominance of a (set of) species by appropriate saddles. Due to the heteroclinic nature of the connections, the dwell time at the saddles is usually much longer than the switching towards the next saddle. For a fractal of RPS games this means that we need recursively defined nested heteroclinic cycles $(\mathrm{HCs})$. On the first (lowest) level the $\mathrm{HC}$ connects three one-species saddle fixed points, representing the temporary dominance of a single species. [In relation to ecology this means that we have to search for microscopic predation rules between $N$ (here

\footnotetext{
${ }^{1}$ It should be noted that our chunking dynamics results from a single set of WLC, while chunking dynamics as discussed in Ref. [11] makes use of additional equations to explicitly implement the hierarchy in timescales.
}

$N=9$ ) species such that three domains play RPS on the coarse level of three metapopulations, while inside the mutually chasing domains, RPS goes on being played between the three species of the corresponding subpopulations.] On the second level, the three saddles are three HCs themselves that are connected by heteroclinic orbits.

This structural hierarchy then generates a hierarchy in timescales if we appropriately control the species trajectory. It will spend a number of revolutions at each $\mathrm{HC}$ on the lower level $n \geqslant 1$ before it turns to the next two HCs on the same level $n$. The full revolution along the cycle on level $n+1$, connecting the former HCs on level $n$, defines the slow timescale.

As we have shown in Ref. [18], the control of the dwell time in the vicinity of saddles or HCs works via a suitable choice of predation rates (based on appropriate conditions on the eigenvalues of the Jacobian [19]). If these hierarchical heteroclinic networks at single sites are then coupled via diffusion on a spatial grid, the hierarchy in timescales should translate to a hierarchy in spatial scales for suitable parameter intervals.

As concrete realization of WLC we choose generalized Lotka-Volterra equations given by

$$
\partial_{t} s_{i}=\delta \nabla_{x}^{2} s_{i}+\rho s_{i}-\gamma s_{i}^{2}-\sum_{j \neq i} A_{i, j} s_{i} s_{j}+\sigma\left|\xi_{i}(t)\right|,
$$

where $s_{i} \equiv s_{i}(x, t) \in \mathbb{R}_{0}^{+}$defines the concentration of species $i \in\{1, \ldots, 9\}$ for two hierarchy levels, $\sigma$ is the noise strength, $\xi_{i}$ is Gaussian white noise with zero mean, $\delta$ is the diffusion rate, and $x$ indicates the location on a two-dimensional spatial grid with $L \times L$ sites. In view of ecological applications, a two-dimensional grid seems reasonable as a first approach. The sites represent patches, each with a well mixed subpopulation of varying occupation number. In view of brain dynamics, consideration of spatially coupled heteroclinic networks is so far lacking. It should be noted that cognitive dynamics in terms of WLC is an effective description, not a description on a neurophysiological level. Thus it seems to be unknown which geometry appropriately describes the spatial coupling between single-site heteroclinic dynamics, where the temporary information items would be located. In this respect, our choice of a two-dimensional grid is one of the simplest possible, but it is already suited to illustrate different options for partial synchronization between the heteroclinic dynamics as we will see.

The numerical integration is carried out via XMDS2, applying the Runge-Kutta fourth-order method and a spectral method for the spatial dimension (for details see $[20,21]$ ). The parameter $\rho$ denotes the reproduction rate, set to one in the following and thereby fixing the timescale; $\gamma$ denotes the death rate. The set $A_{i, j}$ constitutes the predation matrix $A$, chosen as the following block matrix for two hierarchy levels: Its diagonal consists of $3 \times 3$ blocks of the form $m_{1}$, similar to the RPS predation matrix for which $p^{(1)}=1$ and $o^{(1)}=0$. The off-diagonal blocks $p_{2}$ have $p^{(2)}$ on their diagonal and $r$ for the remaining elements; $o_{2}$ is chosen accordingly,

$$
A=\left(\begin{array}{ccc}
m_{1} & p_{2} & o_{2} \\
o_{2} & m_{1} & p_{2} \\
p_{2} & o_{2} & m_{1}
\end{array}\right), \quad m_{1}=\left(\begin{array}{ccc}
0 & p^{(1)} & o^{(1)} \\
o^{(1)} & 0 & p^{(1)} \\
p^{(1)} & o^{(1)} & 0
\end{array}\right) .
$$




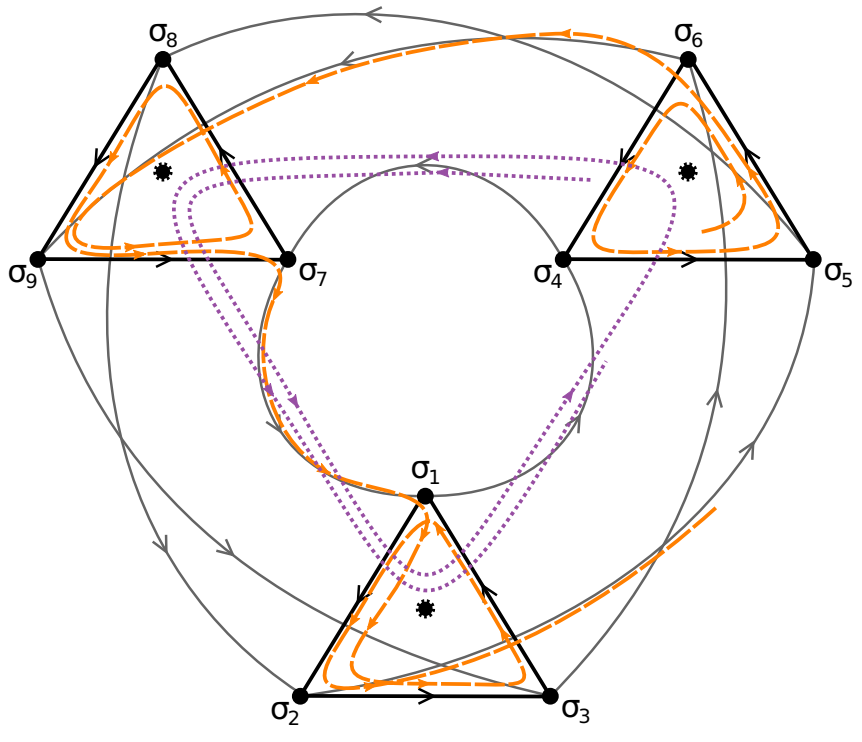

FIG. 1. Schematic view of a heteroclinic network with selected trajectories.

Note the similarity of the block form of $A$ to the form of $m_{1}$. For $n$ intended hierarchy levels, $A$ generalizes to

$$
m_{n}=\left(\begin{array}{ccc}
m_{n-1} & p_{n} & o_{n} \\
o_{n} & m_{n-1} & p_{n} \\
p_{n} & o_{n} & m_{n-1}
\end{array}\right), \quad n=1,2,3, \ldots,
$$

where $m_{0}=0$ and $o_{n}$ and $p_{n}$ are $3^{n-1} \times 3^{n-1}$ matrices, with diagonal elements given by $o^{(n)}, p^{(n)} \in \mathbb{R}$, respectively; $r \in$ $\mathbb{R}$ for the remaining elements. This choice preserves the permutation symmetry within the clusters and between the clusters. The independent parameters are $o^{(n)}, p^{(n)}$, and $r$. To simplify notation in the following, we identify $c=p^{(1)}, d=$ $p^{(2)}, e=o^{(1)}, f=o^{(2)}, g=o^{(3)}, h=p^{(3)}$, and $r$ representing the remaining elements in $p_{2}$ and $o_{2}$.

It should be further noted that this number of independent parameters is dictated by the conditions on the predation rates $(0<e<f<\gamma \wedge e>2 \gamma-e \wedge d>2 \gamma-e \wedge r>\gamma$ for two hierarchy levels). These very conditions are essential for making the (subset of the) heteroclinic network attractive, that is, guaranteeing the time evolution of the trajectory along the prescribed path with a selected $\mathrm{HC}$ of $\mathrm{HCs}$ in the ninedimensional phase space (see [18] for the derivation) and Fig. 1 for such a trajectory.

Figure 1 is schematic. The solid (black and gray) lines are heteroclinic connections within the nine-dimensional phase space. The nodes are the saddles $\sigma_{1}, \ldots, \sigma_{9}$ with two expanding and two contracting heteroclinic connections per saddle as indicated in the figure and one radial and four transversal directions per node (not displayed). We distinguish expanding (contracting) directions towards another saddle within so-called small heteroclinic cycles (SHCs) or within a large heteroclinic cycle (LHC), connecting three SHCs. The dashed trajectory (orange in Fig. 1) shows a typical LHC connecting the three SHCs with at least one revolution within
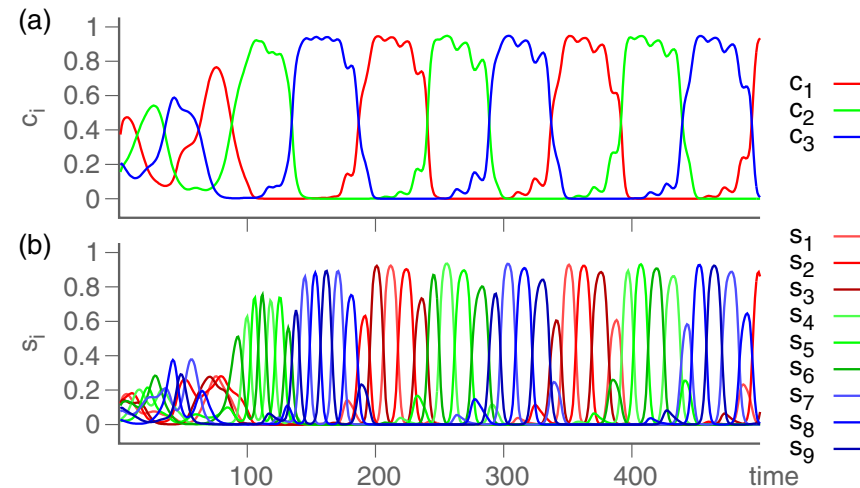

FIG. 2. (a) Cluster activity $c_{i}=\sum_{1 \leqslant j \leqslant 3} s_{3(i-1)+j}$ and (b) time evolution of species concentrations $s_{i}$ near a two-level hierarchical network of nine species, resembling a two-level chunking dynamics under the influence of noise. The parameters are $c=d=2, e=0.2$, $f=0.3, r=1.25, \gamma=1.05$, and $\sigma=10^{-7}$.

each SHC. ${ }^{2}$ The dotted (purple) trajectory follows heteroclinic connections between three-species coexistence fixed points, indicated by the dots in the middle of the triangles.

\section{RESULTS}

\section{A. Heteroclinic network at a single site}

In order to understand the spatial patterns, we first summarize our results for a single hierarchical heteroclinic network [18] and point out how a temporal hierarchy is generated. For a certain range of parameters with $\gamma$ sufficiently small and the difference between parameters $e$ and $f$ sufficiently large, we observe the typical time evolution of an $\mathrm{HC}$ of HCs in the absence of noise: On a short timescale, species 1 chases species 3 , which chases species 2 , which chases species 1 , making up a subpopulation or a cluster of an SHC, and similarly for the other two clusters with three species each. On a larger timescale, it is the three clusters, corresponding to chunks in the sequence of fast oscillating metastable states, which play rock-paper-scissors and modulate the fast oscillations. The resting time at individual saddles and at SHCs increases with the number of revolutions (as visible in Fig. 3) unless there is noise as in Fig. 2, while the motion between different saddles and SHCs happens almost instantaneously (see Fig. 2). Without noise and for three hierarchy levels with 27 species, Fig. 3 demonstrates the slowing down of the periods that is typical for heteroclinic motion without noise; otherwise the time evolution would resemble chunking dynamics on three levels.

The death rate plays the role of a bifurcation parameter. If we tune $\gamma$ to larger values, the system undergoes a sequence

\footnotetext{
${ }^{2}$ Note the self-similar feature of the path in phase space. It corresponds to what is called nested self-similar in contrast to branching self-similar network topology in Ref. [22]. Interestingly, both kinds of topology in ordinary space characteristically differ in their properties of information processing in such networks [22], so one should further explore the implications of nested self-similarity in phase space in view of information processing in the brain.
} 

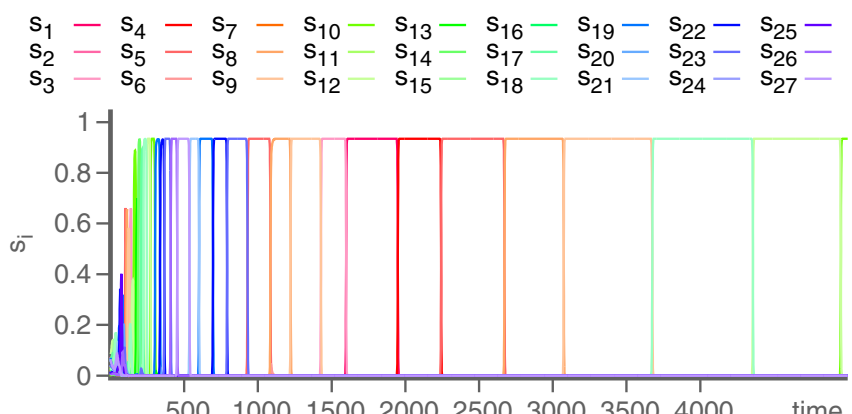

5001000150020002500300035004000 time

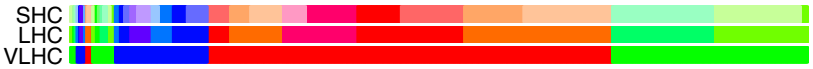

FIG. 3. Time evolution of species concentrations near a threelevel hierarchical heteroclinic network of 27 species. Color bars at the bottom show the closest attractor at a given time, separately for all three levels of hierarchy. The parameters are $c=d=h=2, e=$ $0.18, f=0.23, g=0.38, r=1.25, \gamma=1.08$, and $\sigma=0$.

of Hopf bifurcations. One type happens simultaneously at different cluster coexistence fixed points. When they become attractive, the lowest level of the HC collapses and the remaining LHC now connects the three-species coexistence saddle fixed points. The second type of Hopf bifurcation happens at the global (nine-species) coexistence fixed point. When this fixed point becomes attractive, also the second hierarchy level collapses, corresponding to the slow timescale. The order of bifurcations depends on the choice of the other parameters. For example, for $r=1.25, e=0.2, f=0.3$, and $c=d=2$, two hierarchy levels are observed below $\gamma=1.1$.

In view of generating a temporal hierarchy it is of interest what determines the number of revolutions within the SHCs as this number determines the separation of timescales between the SHCs and LHC(s). The dwell time within an SHC is essentially controlled by how different the two rate parameters $e$ and $f$ are chosen. We have derived an analytical formula for this number of revolutions in an SHC in Ref. [23] via construction of a Poincaré map. Roughly speaking, the larger the difference is, the more revolutions the trajectory spends within each of the three SHCs. For our set of parameters we observed between one and eight orbits within an SHC, leading to a measured ratio of timescales between 3 and 24 .

\section{B. Spatially coupled heteroclinic networks}

\section{Two hierarchy levels}

If we next couple the heteroclinic networks via diffusion according to Eq. (1), we obtain different regimes of pattern formation displayed in Fig. 4. The figure illustrates the effect of the resting time within SHCs on the pattern formation for an intermediate diffusion strength. The left parts of Figs. 4(a)4(c) show the color of the dominant species at each site of the grid with species $1-3,4-6$, and 7-9 in different shades of red, green, and blue, respectively, while the right part focuses on species 1, 2, and 3, mapping their concentration to the intensity of red, green, and blue, respectively. The right part is shown for better resolution of what the dominance of a certain species in the corresponding left panel means. Figure 4(a) refers to the case of nested spirals on the coarse

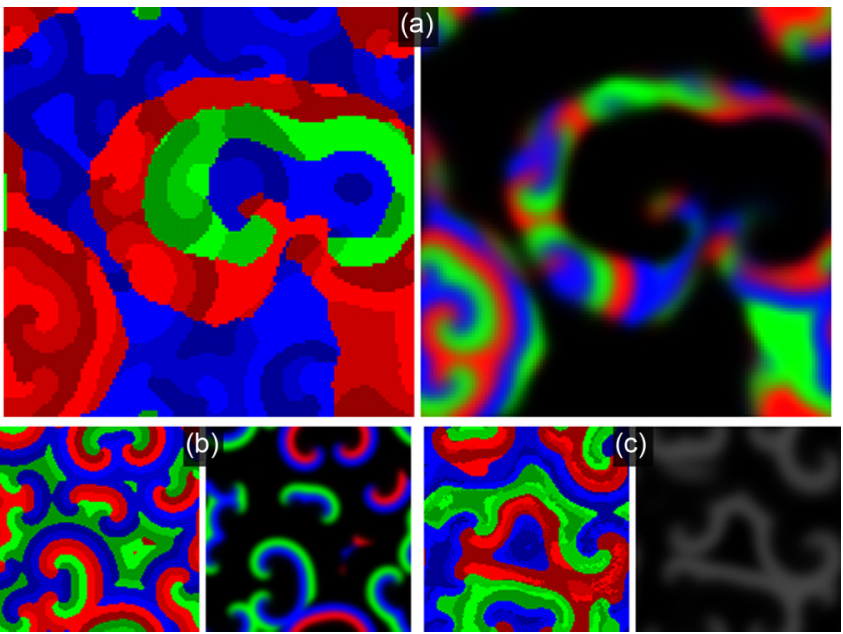

FIG. 4. Spiral patterns as a function of the resting time in SHCs. (a) Nested spirals for $f=0.4, \gamma=0.8$, and $\delta=0.5$ at $t=750$. (b) Six-armed spirals for $f=0.2, \gamma=0.8$, and $\delta=0.5$ at $t=750$. (c) LHC spirals for $f=0.4, \gamma=1.2$, and $\delta=1.0$ at $t=1500$. The remaining parameters are $c=d=2, e=0.2, r=1.25$, and $\sigma=10^{-6}$. The grid size is $128 \times 128$.

scale of the LHC, visible in spiral arms of different color, while different shades of a certain color represent the chasing inside an SHC. The value of the deletion rate $\gamma$ is below the Hopf bifurcation points, so two hierarchy levels are present. The value of $f-e=0.2$ guarantees a number of revolutions within an SHC, thus all shades in the left panel (colors in the right panel) are visible.

Figure 4(b), with the same intermediate value of the diffusion constant and deletion rate but $f-e=0$, shows a case where the resting time in each of the three SHCs is so short that after a visit of two out of three saddles the trajectory turns to the next SHC, so for each SHC only two shades (left panel) (colors, right panel) are visible. A corresponding sequence of visits of the trajectory in Fig. 4(b) would be $\sigma_{4}, \sigma_{5}, \sigma_{8}, \sigma_{9}, \sigma_{3}, \sigma_{1}, \sigma_{4}, \ldots$. This kind of pattern remains for a whole interval of $f-e$ values, up to $f-e=0.06$ for the chosen parameters.

In Fig. 4(c) the lower hierarchy level has collapsed because of the choice of $\gamma$ above the (second) Hopf bifurcation point at which the three-species coexistence fixed point becomes stable. Only LHC spirals (i.e., spirals of the clusters) are seen. The LHC connects the three three-species coexistence fixed points. As becomes clear from the right panel of Fig. 4(c), the random shades in the left panel are artifacts of the color coding, as the gray in the right panel reflects the superposition of red, green and blue, representing the mixing of species 1-3 at the coexistence fixed points at all sites of the grid.

Figure 5 presents the role of diffusion for nested spiral formation. The difference between the upper and lower panels is the same as between the left and right panels of Fig. 4. Here, from left to right, the diffusion strength increases: In Fig. 5(a) the diffusion strength is sufficiently small so that the coexisting large spiral arms fit onto the grid. For stronger diffusion [Figs. 5(b) and 5(c)], only one arm of the larger spirals fits onto the grid with species of the red SHC chasing each other at time $t=750$ [Fig. 5(b)] until the SHC suddenly 

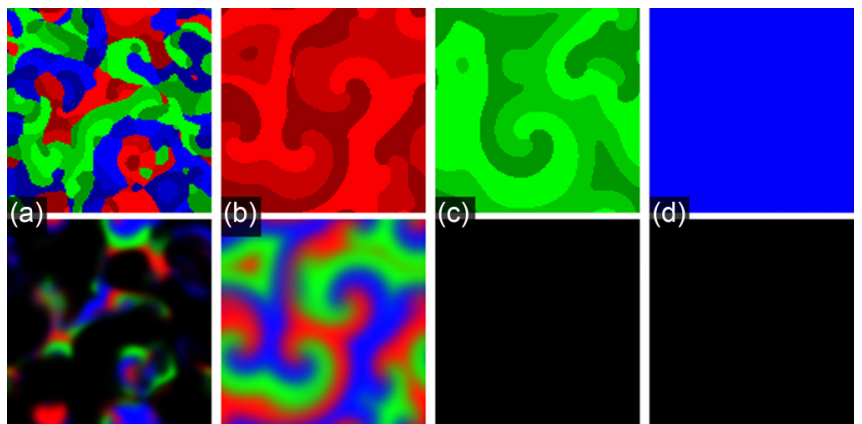

FIG. 5. Influence of increasing diffusion on patterns of nested spirals. (a) Nested spirals ( $\delta=0.5$ at time $t=750$ ). (b) and (c) SHC spirals $(\delta=2.0)$ at times (b) $t=750$ and (c) $t=1000$. (d) Uniform state with only species $2(\delta=10$ and $t=750)$. The remaining parameters are $c=d=2, e=0.2, f=0.3, r=1.25, \gamma=0.8$, and $\sigma=0$ on a $128 \times 128$ grid.

switches to the green one [Fig. 5(c) at time $t=1000]$ and subsequently to the blue one (not displayed). Note that this switching occurs without noise, but due to the heteroclinic connection, and shows the typical slowing down of heteroclinic connections that would be avoided by additive noise. In both Figs. 5(b) and 5(c), the LHCs have synchronized towards the same SHC on all sites of the given grid, while inside an SHC the competition continues between species on different grid sites. The black color in the lower panel of Fig. 5(c) just indicates the absence of the first three species at $t=1000$. At even stronger diffusion [Fig. 5(d)], the species concentrations also inside an SHC have fully synchronized. The uniform blue shade of the upper panel corresponds to species 9, the black color in the lower panel of Fig. 5(d) indicates again the absence of the first three species. The further time evolution of the upper panel of Fig. 5(d) will be an almost instantaneous flip first towards different uniform shades of red, followed by flips between different uniform shades of green and later blue, until a full revolution of an LHC is completed and starts again. Here, just as in Figs. 5(b) and 5(c), noise would prevent the slowing down of this switching.

Note in particular the order of synchronization: First the heteroclinic motion synchronizes towards the same SHC of an LHC and then towards temporary resting at the same saddles. The reason is that diffusion acts on all sites in the bulk as well as predator-prey interaction between species of the same SHCs, while predator-prey interactions between species of different SHCs are only confronted via diffusion at interfaces between large spiral arms. Therefore, diffusion is effectively stronger with respect to predation processes at interfaces than with respect to those in the bulk and supports the synchronization accordingly.

It should be noted that the partial or full synchronization of heteroclinic orbits on the spatial grid amounts to a large dimensional reduction of phase space. It will be very interesting to explore the implications of synchronization patterns on heteroclinic networks that are spatially coupled and organized in neuronal networks. In brain dynamics it is known that processes effectively reduce to low-dimensional dynamics [8] in spite of the high-dimensional phase space that is in principle available.

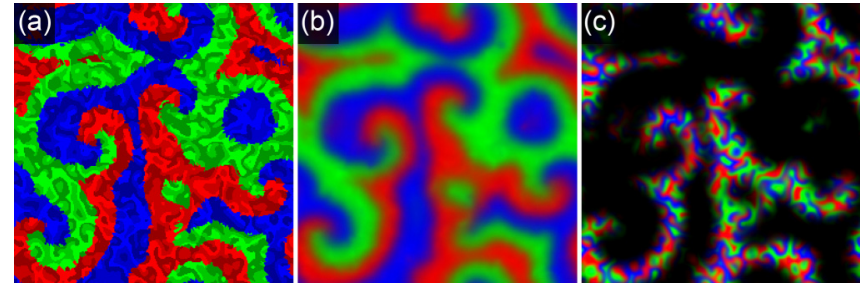

FIG. 6. Pattern formation again for a two-level system here with enhanced cluster diffusion for comparison. Due to cluster diffusion, the spatial structures get resolved in more detail. (a) Dominating species at each site. Also shown are the concentrations of (b) clusters $c_{1}, c_{2}$, and $c_{3}$ and (c) species $s_{1}, s_{2}$, and $s_{3}$, coded as RGB color values. The parameters are $c=d=2, e=0.2, f=0.3, r=1.25$, $\gamma=0.9, \sigma=0, \eta=2.0, \delta=0.1, \theta=0, L=256$, and $t=2000$.

We have verified that the results are independent of the choice of the numerical algorithm and the step size (see [21]). Also, additive noise implemented as in Ref. [18] has little influence on the resulting patterns [21].

\section{Three hierarchy levels and enhanced cluster diffusion}

We turn to the case of three hierarchy levels. Following the construction principle via an appropriate choice of eigenvalues, we choose the predation matrix according to Eq. (3) with the additional parameters $g=o^{(3)}$ and $h=p^{(3)}$. For a broad range of parameters, the time evolution of the 27 species shows the characteristics of an $\mathrm{HC}$ of an $\mathrm{HC}$ of an $\mathrm{HC}$ of three single-species saddles, as we have seen in Fig. 3, thus chunking dynamics with three hierarchy levels. However, the attempt to visualize three levels of a fractal spatial structure of spirals on a moderate lattice size is hindered by the fact that the spirals on the lowest level need enough space to unfold within the arms of other spirals. While our tuning of timescale separation by means of $(f-e)$ leads to a slight increase in the size of spiral arms that represent one SHC, the addition of nonlinear diffusion terms additionally supports the separation of spatial scales. We add to the diffusion of Eq. (1) the terms

$$
\left(s_{i} / c_{i}\right) \eta \nabla_{x}^{2} c_{i}+\left(s_{i} / d_{i}\right) \theta \nabla_{x}^{2} d_{i},
$$

with

$$
c_{i}=s_{i}+s_{e s(i)}+s_{c s(i)} \forall i \in\{1, \ldots, 27\}
$$

and

$$
\begin{aligned}
d_{i}= & s_{i}+s_{e s(i)}+s_{c s(i)}+s_{e l(i)}+s_{e s(e l(i))} \\
& +s_{c s(e l(i))}+s_{c l(i)}+s_{e s(c l(i))}+s_{c s(c l(i)),}
\end{aligned}
$$

where the indices represent the expanding $(e)$ and contracting $(c)$ directions in the small $(s)$ and large $(l)$ heteroclinic connections, respectively, $\eta$ and $\theta$ are parameters to tune the relative strength of the two terms. This choice amplifies the common diffusion of species, belonging to the same cluster or supercluster of species, respectively. Figure 6 shows a better resolution of the spatial structures for two hierarchy levels, while Fig. 7 represents the case of three hierarchy levels, where Figs. 7(b) and 7(c) correspond to zooms into the first group on the upper hierarchy level. Physically, the extra nonlinear diffusion terms would mean that species belonging to the same cluster of a population or supercluster 

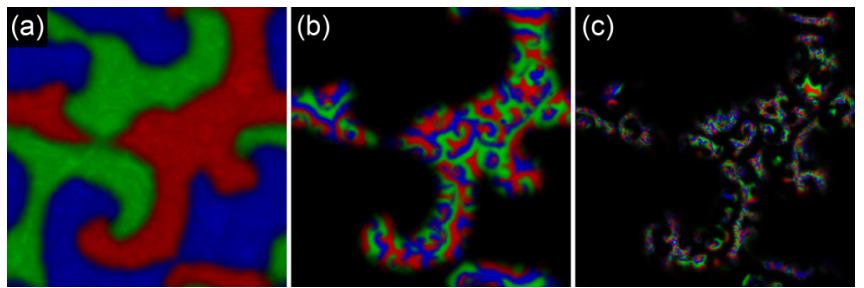

FIG. 7. Three levels of nested spirals with enhanced cluster diffusion. Red codes species (a) 1-9, (b) 1-3, and (c) 1; green (a) 10-18, (b) 4-6, and (c) 2; and blue (a) 19-27, (b) 7-9, and (c) 3 to illustrate the RPS game played on each scale. The parameters are $c=d=h=2, e=0.2, f=0.4, g=0.6, \gamma=0.9, r=1.25$, $\delta=0.01, \eta=0.1, \theta=1.0$, and $\sigma=10^{-6}$.

of a metapopulation experience an additional drive to move collectively, apart from the bulk tendency of diffusion.

\section{CONCLUSION}

In summary, we have demonstrated how to construct a hierarchical attractor with single-species saddles on the basic level and iterated heteroclinic connections between them. Directing the trajectory in this high-dimensional phase space along selected heteroclinic network connections allows for varying the temporary alliances and preselecting the competing partners. For the implementation on a spatial grid we have chosen the simplest way of attractive interaction via diffusion. Diffusion translates the timescale separation to nested spirals on spatial grids. For sufficiently strong diffusion this pattern formation is a transient phenomenon, before the heteroclinic motion on all sites of the finite grid synchronizes, so the patterns disappear, though the cycling continues uniformly at all sites. For future work it would be interesting to study other types of spatial coupling of heteroclinic networks, each assigned to a single site, and analyze their impact on synchronization, dimensional reduction, and the induced pattern formation, in particular in view of brain dynamics.

\section{ACKNOWLEDGMENTS}

We would like to thank Darka Labavić (University of Lille) for valuable discussions in the beginning of this project and the group of M. Pleimling for discussions during our stay at Virginia Tech. Financial support from the German Research Foundation (DFG, Grants No. ME-1332/27-1 and No. ME$1332 / 28-1)$ is gratefully acknowledged.
[1] A. A. Fingelkurts and A. A. Fingelkurts, Information 8, 22 (2017).

[2] D. Vidaurre, S. M. Smith, and M. W. Woolrich, Proc. Natl. Acad. Sci. USA 114, 12827 (2017).

[3] F. E. Turkheimer, R. Leech, P. Expert, L.-D. Lord, and A. C. Vernon, Neurosci. Biobehav. Rev. 55, 211 (2015).

[4] D. La Rocca, N. Zilber, P. Abry, V. van Wassenhove, and P. Ciuciu, J. Neurosci. Methods 309, 175 (2018).

[5] O. Sporns, Biosystems 85, 55 (2006).

[6] C. M. Michel and T. Koenig, Neuroimage 180, 577 (2018).

[7] M. I. Rabinovich, P. Varona, A. I. Selverston, and H. D. I. Abarbanel, Rev. Mod. Phys. 78, 1213 (2006).

[8] M. I. Rabinovich and P. Varona, Front. Comput. Neurosci. 12, 73 (2018).

[9] V. Afraimovich, I. Tristan, R. Huerta, and M. I. Rabinovich, Chaos 18, 043103 (2008).

[10] D. Van de Ville, J. Britz, and C. M. Michel, Proc. Natl. Acad. Sci. USA 107, 18179 (2010).

[11] M. I. Rabinovich, P. Varona, I. Tristan, and V. Afraimovich, Front. Comput. Neurosci. 8, 22 (2014).

[12] A. Szolnoki, M. Mobilia, L.-L. Jiang, B. Szczesny, A. M. Rucklidge, and M. Perc, J. R. Soc. Interface 11, 20140735 (2014).
[13] U. Dobramysl, M. Mobilia, M. Pleimling, and U. C. Täuber, J. Phys. A: Math. Theor. 51, 063001 (2018).

[14] G. Szabó, A. Szolnoki, and I. Borsos, Phys. Rev. E 77, 041919 (2008).

[15] A. Roman, D. Konrad, and M. Pleimling, J. Stat. Mech. (2012) P07014.

[16] B. L. Brown and M. Pleimling, Phys. Rev. E 96, 012147 (2017).

[17] D. Labavić and H. Meyer-Ortmanns, J. Stat. Mech. (2016) 113402.

[18] M. Voit and H. Meyer-Ortmanns, Eur. Phys. J. Spec. Top. 227, 1101 (2018)

[19] P. Ashwin and C. Postlethwaite, Physica D 265, 26 (2013).

[20] G. R. Dennis, J. J. Hope, and M. T. Johnsson, Comput. Phys. Commun. 184, 201 (2013).

[21] See Supplemental Material at http://link.aps.org/supplemental/ 10.1103/PhysRevResearch.1.023008 for details.

[22] S. DeDeo and D. C. Krakauer, J. R. Soc. Interface 9, 2131 (2012).

[23] M. Voit and H. Meyer-Ortmanns, Appl. Math. Nonl. Sci. 4, 279 (2019). 